\title{
Investigation of binary compounds using electron Rutherford backscattering
}

\author{
M. R. Went ${ }^{\mathrm{a})}$ and M. Vos \\ Atomic and Molecular Physics Laboratories, Research School of Physical Sciences and Engineering, The \\ Australian National University, Canberra, Australian Capital Territory 0200, Australia
}

(Received 27 November 2006; accepted 15 January 2007; published online 13 February 2007)

\begin{abstract}
High-energy $(40 \mathrm{keV})$ electrons, scattering over large angles, transfer a small fraction of their kinetic energy to the target atoms, in the same way as ions do in Rutherford backscattering experiments. The authors show here that this energy transfer can be resolved and used to determine the mass of the scattering atom. In this way information on the surface composition for thicknesses of the order of $10 \mathrm{~nm}$ can be obtained. The authors refer to this technique as "electron Rutherford backscattering." In addition the peak width reveals unique information about the vibrational properties (mean kinetic energy) of the scattering atoms. Here the authors demonstrate that the method can be used to identify a number of technologically important compounds. (C) 2007 American Institute of Physics. [DOI: 10.1063/1.2535986]
\end{abstract}

As early as 1967 , Boersch et al. ${ }^{1}$ showed that the energy of $\mathrm{keV}$ electrons scattered elastically over large angles is reduced by the recoil energy $E_{r}$. For scattering from a stationary atom (mass $M$ ) the magnitude of $E_{r}$ is determined by the momentum transfer $\mathbf{q}$ of the electron to the atom: $E_{r}=q^{2} / 2 M$. In general the atoms in a solid will be vibrating (even at $0 \mathrm{~K}$ one has to consider the zero-point motion) and this leads to Doppler broadening of $E_{r}$. For vibrating atoms $E_{r}$ is given by

$$
E_{r}=\frac{q^{2}}{2 M}+\frac{\mathbf{q} \cdot \mathbf{p}}{M},
$$

where $\mathbf{p}$ is the momentum of the target atom before the collision. Thus the naive picture, that the incident and the final energy of the scattered electron are the same has to be replaced by the notion that the sum of the kinetic energies of the atom and electron is conserved. In the context of neutron scattering Paoli and Holt $^{2}$ showed that a simple relation exists between the width of the elastic peak $(\sigma)$ and the mean kinetic energy of the atoms $\left(\bar{E}_{k}\right)$ :

$$
\sigma=\sqrt{\frac{4}{3} \bar{E}_{k} \bar{E}_{r}},
$$

where $\bar{E}_{r}=q^{2} / 2 M$ is the mean recoil energy. Consider a compound containing a number of elements $A, B, C$, etc. Equation (1) implies that, given sufficient energy resolution, the spectrum of elastically scattered electrons will consist of a number of individual peaks appearing at slightly different energy losses, each peak corresponding to a constituent element. Further, the ratio of the intensity of these peaks will be directly related to the elastic cross section $d \sigma / d \Omega$ and the relative density of the atoms within a sample, $N_{A}: N_{B}: N_{C}: \cdots$. The relative peak areas are given by

$$
N_{A} \frac{d \sigma_{A}}{d \Omega}: N_{B} \frac{d \sigma_{B}}{d \Omega}: N_{C} \frac{d \sigma_{C}}{d \Omega}: \cdots .
$$

Thus the technique provides a convenient method for determining the sample composition if the cross sections are known or, if the composition is known, a method by which

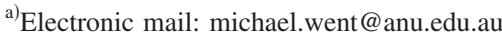

the ratio of the differential cross sections can be determined. This is only true for homogeneous samples. For layered samples inelastic scattering in the overlayer of electrons elastically scattered in the underlying layers changes the ratio depending on the thickness and inelastic mean free path (IMFP) of the overlayer. ${ }^{3}$

In electron Rutherford backscattering (ERBS), just as in photoemission, the information is contained in the electrons that have not lost energy due to inelastic scattering (i.e., electronic excitations). Energy loss due to inelastic scattering is almost always much larger than the separation of the elastic peaks, and after inelastic scattering it will generally not be possible to determine the nature of the scatterer. The depth that is sampled is determined by the IMFP and the measurement geometry. This is in contrast to Rutherford backscattering (RBS), where depth information is obtained from the amount of energy lost due to inelastic excitations ("electronic stopping").

This technique, employing a scattering angle $\theta \approx 45^{\circ}$, has been used to examine the kinetic energy of carbon atoms in graphite $^{4}$ and to determine the IMFP in amorphous carbon. ${ }^{3}$ Very recently, an additional electron gun (Kimball Physics ELG-2 with a $\mathrm{BaO}$ filament, for reduced thermal spread of the beam) was added at $\theta \approx 120^{\circ}$ (Fig. 1). The larger scattering angle $\left[q=2 k_{0} \sin (\theta / 2)\right.$ with $k_{0}$ the electron momentum] improves the separation of different elements

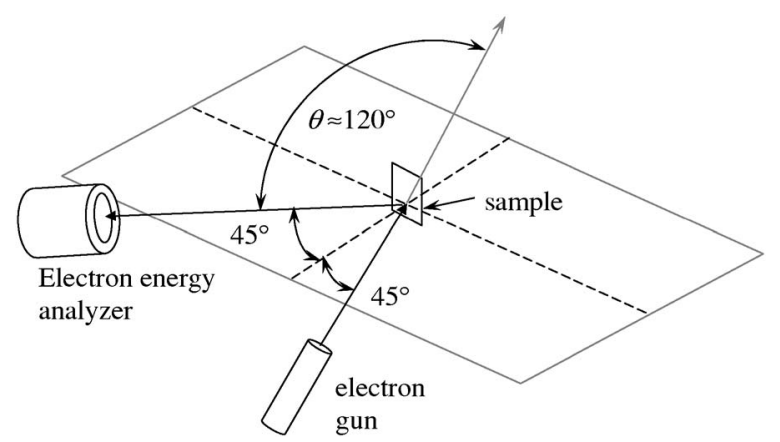

FIG. 1. Schematic diagram showing the relative angles of the electron gun, sample, and electron energy analyzer. The electron gun is mounted $45^{\circ}$ below the horizontal plane; the electron energy analyzer is mounted at $45^{\circ}$ with respect to the vertical plane containing the gun. 


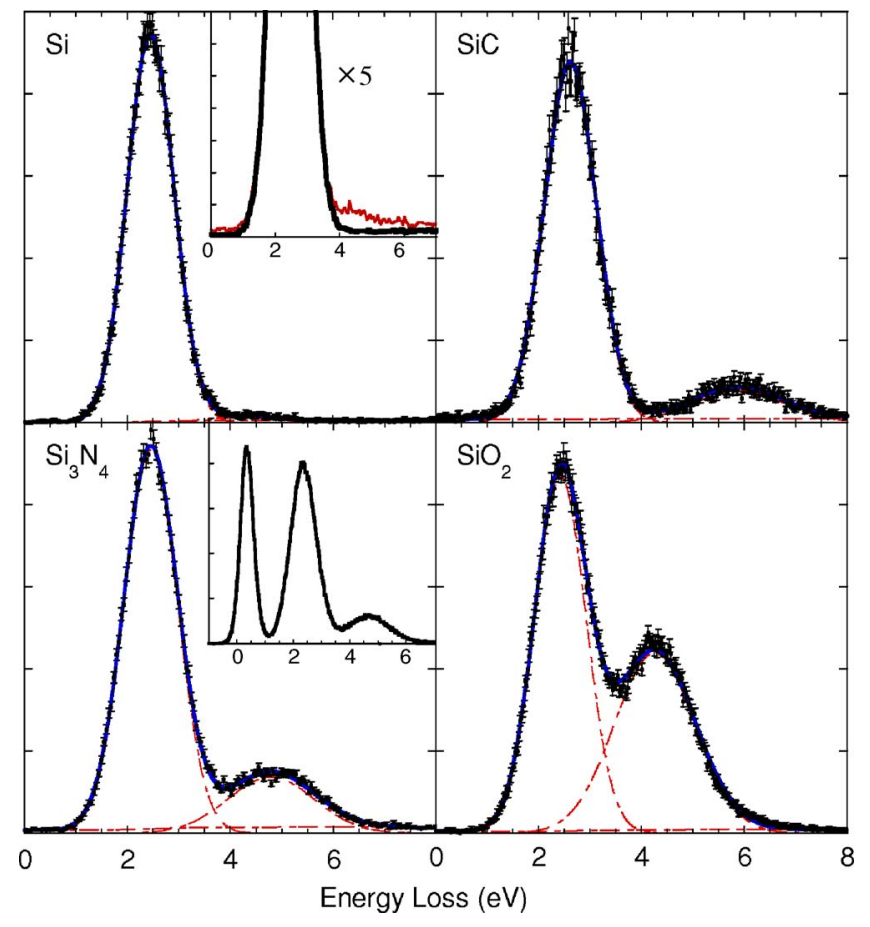

FIG. 2. (Color online) ERBS measurements of Si and Si compounds. The measurements show the clear elastic peak separation possible with this technique. The Si inset shows the Si spectrum before (thin) and after (thick) ion sputtering. The sputtering removed some $\mathrm{C}$ and $\mathrm{O}$ contamination from the surface. The $\mathrm{Si}_{3} \mathrm{~N}_{4}$ spectrum shows as an inset the result of Au evaporation on this film. Now a third, narrower peak is visible at smaller loss values due to $\mathrm{Au}$.

greatly [see Eq. (1)]. The gun emits $500 \mathrm{eV}$ electrons impinging on a sample mounted in the center of the scattering region which is at $39.5 \mathrm{kV}$. Thus $40 \mathrm{keV}$ electrons hit the sample. Those scattered over $\approx 120^{\circ}$ are focused and decelerated by slit lenses in front of a hemispherical analyzer operating at $200 \mathrm{eV}$ pass energy. A position sensitive detector allows for simultaneous measurement of electrons over a range of energies $(\approx 40 \mathrm{eV})$. For a more detailed explanation of the analyzers, see Ref. 5.

In Fig. 2 we show spectra (obtained in less than $1 \mathrm{~h}$, using a beam current of $3 \mathrm{nA}$ ) of the elastic peak of $\mathrm{Si}$, single crystal $\mathrm{SiC}$, and thin layers $(\approx 100 \mathrm{~nm})$ of $\mathrm{SiO}_{2}$ and $\mathrm{Si}_{3} \mathrm{~N}_{4}$. The $\mathrm{SiO}_{2}$ and $\mathrm{Si}_{3} \mathrm{~N}_{4}$ were prepared by plasma enhanced chemical vapor deposition on a silicon substrate. The stoichiometry of $\mathrm{SiO}_{2}$ was confirmed using conventional RBS. The $\mathrm{Si}_{3} \mathrm{~N}_{4}$ samples were too small to check in this manner. The use of thin films circumvents problems due to excessive charging of the sample surface for insulators. The $\mathrm{Si}$ sample was measured before and after sputter cleaning, but the difference was minor (Fig. 2 inset). Due to the high kinetic energies these experiments are only moderately surface sensitive, and the effect of surface cleaning is small. Generally the data presented here are obtained without sputter cleaning. The spectra of the compounds show wellseparated peaks, with varying separation and intensity ratio. From Table I it is clear that the agreement between calculated and measured peak separations is good.

In Table I we also present the theoretical and calculated peak areas for the three silicon compounds. Rutherford cross sections as well as cross sections obtained from the ELSEPA $\operatorname{program}^{6}$ are given. The Rutherford cross section is for scattering from a bare nucleus and is proportional to $Z^{2}$. The ELSEPA cross section takes the effect of screening of the nuclear charge by the electrons into account. The effect of screening is obviously small for these lighter elements. Qualitatively the observed intensity ratio is reproduced by the calculated one: it increases strongly going from $\mathrm{SiC}$ to $\mathrm{SiO}_{2}$. Quantitatively the measured intensity ratio deviates from the calculated one by up to $20 \%$, whereas the experimental error and reproducibility is estimated to be $\approx 10 \%$. The reproducibility is better than $10 \%$.

Comparing the width of the Au peak (Fig. 2 inset) with the Si peak in the four spectra, it is clear that the measured $\sigma$ (450 meV for $\mathrm{Si}$ and $500 \mathrm{meV}$ for the compounds) is considerably larger than that of the Au peak $(250 \mathrm{meV})$. The width of the gold peak is taken as an estimate of the experimental resolution (energy spread of incoming beam and analyzer resolution) as the Doppler broadening of $\mathrm{Au}$ is negligible because of its large mass [see Eq. (1)]. As such we attribute the additional width of the Si peaks to the kinetic energy of the Si atoms in the crystal lattice. Applying Eq. (2) we determine the mean kinetic energy of $\mathrm{Si}$ atoms to be $43 \pm 5 \mathrm{meV}$ in a Si lattice but to be $58 \pm 5 \mathrm{meV}$ when in $\mathrm{SiC}$, $\mathrm{Si}_{3} \mathrm{~N}_{4}$, and $\mathrm{SiO}_{2}$.

In order to show that this technique still works for heavier compounds, in spite of a decrease in separation in energy, we studied InP and $\mathrm{MoS}_{2}$. Results are shown in Fig. 3, as well as Table I. Again the splitting of the peak is well described by the theory. Deviations between the Rutherford cross section and the ELSEPA cross section is now more substantial, and the experiment agrees more with the latter. However, for $\mathrm{InP}$ the observed intensity ratio deviates still from the calculated (ELSEPA) one by $20 \%$. In spite of the fact that the P peak is rather weak compared to the In peak, which makes a determination of the peak ratio difficult, we are surprised about the size of this discrepancy.

A possible origin of this deviation could be an In enrichment of the surface. To investigate this the sample was sputtered with $800 \mathrm{eV} \mathrm{Ar}^{+}$ions. Subsequent ERBS measurement showed no significant change in the intensity ratio of the In

TABLE I. Measured and calculated values for the peak intensity ratios, peak separations, and Gaussian peak width for the binary compounds investigated. Rutherford cross sections and peak intensity ratios are presented for comparison with those calculated by the ELSEPA program of Salvat $e t$ al. (Ref. 6).

\begin{tabular}{|c|c|c|c|c|c|c|c|c|c|c|c|}
\hline \multirow{2}{*}{$\begin{array}{l}\text { Compound } \\
\qquad A_{x} B_{y}\end{array}$} & \multicolumn{3}{|c|}{ Rutherford } & \multicolumn{3}{|c|}{ Salvat et al. (Ref. 6) } & \multirow{2}{*}{$\begin{array}{l}I_{A} / I_{B} \\
\text { Meas. }\end{array}$} & \multicolumn{2}{|c|}{ Separation } & \multirow{2}{*}{$\begin{array}{l}\sigma_{A} \\
(\mathrm{me}\end{array}$} & \multirow{2}{*}{$\begin{array}{l}\sigma_{B} \\
\mathrm{~V})\end{array}$} \\
\hline & $d \sigma_{A} / d \Omega\left(\mathrm{cm}^{2} / \mathrm{sr}\right)$ & $d \sigma_{B} / d \Omega\left(\mathrm{cm}^{2} / \mathrm{sr}\right)$ & $x \sigma_{A} / y \sigma_{B}$ & $d \sigma_{A} / d \Omega\left(\mathrm{cm}^{2} / \mathrm{sr}\right)$ & $d \sigma_{B} / d \Omega\left(\mathrm{cm}^{2} / \mathrm{sr}\right)$ & $x \sigma_{A} / y \sigma_{B}$ & & Calc. & Meas. & & \\
\hline $\mathrm{SiC}$ & $3.04 \times 10^{-22}$ & $5.59 \times 10^{-23}$ & 5.4 & $2.85 \times 10^{-22}$ & $5.04 \times 10^{-23}$ & 5.7 & 6.6 & 3.27 & 3.27 & 500 & 860 \\
\hline $\mathrm{Si}_{3} \mathrm{~N}_{4}$ & $3.04 \times 10^{-22}$ & $7.61 \times 10^{-23}$ & 3.0 & $2.85 \times 10^{-22}$ & $6.88 \times 10^{-23}$ & 3.1 & 4.2 & 2.41 & 2.30 & 520 & 880 \\
\hline $\mathrm{SiO}_{2}$ & $3.04 \times 10^{-22}$ & $9.94 \times 10^{-23}$ & 1.5 & $2.85 \times 10^{-22}$ & $9.02 \times 10^{-23}$ & 1.6 & 1.4 & 1.85 & 1.83 & 500 & 700 \\
\hline $\mathrm{InP}$ & $3.73 \times 10^{-21}$ & $3.49 \times 10^{-22}$ & 11 & $4.90 \times 10^{-21}$ & $3.28 \times 10^{-22}$ & 15 & 18 & 1.60 & 1.50 & 250 & 470 \\
\hline $\mathrm{MoS}_{2}$ & $2.74 \times 10^{-21}$ & $3.98 \times 10^{-22}$ & 3.4 & $3.33 \times 10^{-21}$ & $3.77 \times 10^{-22}$ & 4.4 & 4.6 & 1.41 & 1.40 & 280 & 440 \\
\hline
\end{tabular}




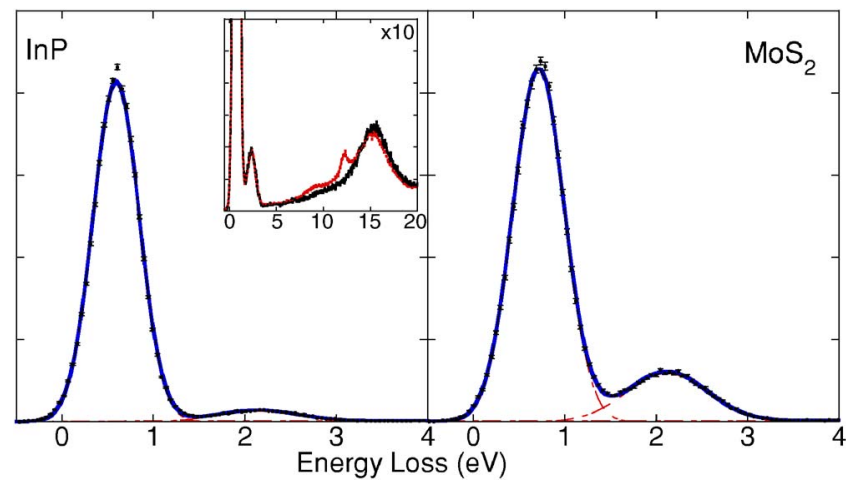

FIG. 3. (Color online) (Left) ERBS spectra of an InP wafer. Inset shows the extended energy loss spectra for the sample before (solid, black) and after (dashed, red) sputtering. The In plasmon that appears after sputtering shows that In islands are formed, however, the $\mathrm{In} / \mathrm{P}$ peak ratio change is minor. (Right) ERBS spectra of a $\mathrm{MoS}_{2}$ crystal.

and $\mathrm{P}$ peaks, again indicating that the surface sensitivity of this technique is rather mild. However, by examining the spectra over a larger energy range (Fig. 3 inset), we see the appearance of extra structures at $\approx 8 \mathrm{eV}$ and $\approx 12 \mathrm{eV}$. At this energy loss range electronic excitations have occurred in addition to the elastic scattering event. These energy loss values correspond to the surface and bulk plasmon energy of pure In metal. This is not unexpected as InP is known to form In islands on the surface, as the more reactive $\mathrm{P}$ atoms are removed by sputtering. ${ }^{7,8}$

We have demonstrated that scattering of $\mathrm{keV}$ electrons can provide information on the sample composition in a way similar to conventional RBS. For an electron spectroscopy ERBS is rather bulk sensitive, probing the sample up to a depth of several nanometers. Peak separation is predicted with an accuracy of $0.1 \mathrm{eV}$ (in spite of a nominal resolution of $0.5 \mathrm{eV}$ ), but for the peak area ratio, deviations with theory of up to $20 \%$ exist. A possible explanation would be that the calculated cross sections are incorrect, as these have not been tested under these conditions. However, this seems unlikely, as for the lighter elements the Rutherford cross section is similar to the ELSEPA one, indicating that the screening effects are minor. Under these experimental conditions different choices of the correlation-polarization potential have very limited effect on the obtained cross sections. ${ }^{9,10}$

In the analysis of these measurements it is assumed that a single scattering approach may be used. This assumption has been shown to be valid for a similar class of problems at much lower energies $(<2 \mathrm{keV})$ for targets in the same mass range $(Z<47){ }^{11}$ and it is difficult to see how this would not be true for the current measurement.

In spite of this it is clear that large-angle scattering of energetic electrons can provide valuable information on the composition in the near surface area. This provides exciting opportunities, especially if these experiments could be done in combination with good lateral resolution, using focused electron beams as are available in scanning electron microscopes.

The authors would like to thank L. Chadderton and M. Fraser for providing the samples used in this work and R. G. Elliman for checking the stoichiometry of $\mathrm{SiO}_{2}$ using conventional RBS. This work is made possible by a grant of the Australian Research Council.

${ }^{1}$ H. Boersch, R. Wolter, and H. Schoenebeck, Z. Phys. 199, 124 (1967).

${ }^{2}$ M. P. Paoli and R. S. Holt, J. Phys. C 21, 3633 (1988).

${ }^{3}$ M. Went, M. Vos, and R. G. Elliman, J. Electron Spectrosc. Relat. Phenom. (in press).

${ }^{4}$ M. Vos and M. Went, Phys. Rev. B 74, 205407 (2006).

${ }^{5}$ M. Vos, G. P. Cornish, and E. Weigold, Rev. Sci. Instrum. 71, 3831 (2000).

${ }^{6}$ F. Salvat, A. Jablonski, and C. Powell, Comput. Phys. Commun. 165, 157 (2005).

${ }^{7}$ O. Wada, J. Phys. D 17, 2429 (1984).

${ }^{8}$ F. Stietz, T. Allinger, V. Polyakov, J. Woll, A. Goldmann, W. Erfurth, G. Lapeyre, and J. Schaefer, Appl. Surf. Sci. 104/105, 169 (1996).

${ }^{9}$ A. Jablonski, F. Salvat, and C. Powell, Surf. Interface Anal. 37, 1115 (2005).

${ }^{10}$ F. Salvat, Phys. Rev. A 68, 012708 (2003).

${ }^{11}$ V. M. Dwyer, J. Vac. Sci. Technol. A 12, 2680 (1994). 